\title{
Gender and Age at Enrolment: the Predictor of Advanced Heart failure in Gene-negative Patients with Hypertrophic Cardiomyopathy
}

Lin Qi

The Affiliated Hospital of Qingdao University https://orcid.org/0000-0001-7199-0679

\section{Zhengyi Shan}

The Affiliated Hospital of Qingdao University

\section{Ying Wei}

The Affiliated Hospital of Qingdao University

\section{Qing Yao Wang}

The Affiliated Hospital of Qingdao University

Peng Zhao ( $\nabla$ qyzhaopeng@sina.com )

The Affiliated Hospital of Qingdao University https://orcid.org/0000-0002-8521-3894

\section{Research}

Keywords: Hypertrophic cardiomyopathy, gene-negative, gender, age at enrolment, advanced heart failure

Posted Date: June 15th, 2021

DOI: https://doi.org/10.21203/rs.3.rs-596888/v1

License: (9) This work is licensed under a Creative Commons Attribution 4.0 International License. Read Full License 
Gender and Age at Enrolment: The Predictor of Advanced Heart failure in Gene-negative Patients with Hypertrophic Cardiomyopathy

Lin Qi ${ }^{1}$, Zhengyi Shan ${ }^{1}$, Ying Wei ${ }^{2}$, Qingyao Wang ${ }^{1}$, Peng Zhao ${ }^{1}$

${ }^{1}$ Department of Pathology, The Affiliated Hospital of Qingdao University, Qingdao, China

${ }^{2}$ Operating room, The Affiliated Hospital of Qingdao University, Qingdao, China

The corresponding author: Dr. Peng Zhao, Department of Pathology, The Affiliated Hospital of Qingdao University, No. 16 Jiangsu Road, Qingdao 266003, China. e-mail: qyzhaopeng@sina.com.

\begin{abstract}
BACKGROUND: Patients with hypertrophic cardiomyopathy (HCM) may develop concomitant advanced heart failure (HF). However, there is a paucity of data on the clinical outcomes of HCM patients without mutations who have advanced HF.
\end{abstract}

Methods: A total of 1529 unrelated patients with HCM were enrolled and followed up. All patients were genotyped by whole exome or panel sequencing. Patients without mutations were studied to assess the impact of family history, clinical findings and echocardiographic parameters on advanced HF.

Results: A total of 735 unrelated patients with HCM were included in the study. The mean follow up duration was $3.2 \pm 2.3$ years. During follow-up,97 patients had advanced HF. Multivariable analysis revealed that risk factors significantly associated with advanced HF were gender [adjusted hazard ratio (HR)2.499, 95\% confidence interval (CI) 1.531-4.081, $\mathrm{P}<0.001$ ] and age at enrolment (adjusted HR 1.298,95\% CI 1.00-1.682, $\mathrm{P}=0.049$ ) during the follow-up period.

Conclusion: Female and older at enrolment can increase risk of advanced HF in gene-negative patients with HCM. Early detection and treatment have an important role to play in management and prevention of disease-related complications for gene-negative patients.

Keywords: Hypertrophic cardiomyopathy; gene-negative; gender; age at enrolment; advanced heart failure 
Hypertrophic cardiomyopathy (HCM) is a complex type of genetic heart disease that is defined by left ventricular hypertrophy unexplained by secondary causes[1].Over the past 20 years, a lot of evidence has supported the prevalence of $\mathrm{HCM}$ at about 1/500 USA[2], as well as in China[3].However, recent studies have shown that HCM is more common than previously estimated, with an estimated prevalence ranging from 1 in 500 to 1 in 200[4].

The demographics and epidemiology of HCM have changed, and there is increasing recognition of the dysfunction caused by exertion dyspnea, which has traditionally been considered a form of heart failure (HF) [5, 6]. With the decrease in sudden cardiac death (SCD) caused by the use of implantable defibrillators in $\mathrm{HCM}, \mathrm{HF}$ has become an increasingly prominent management problem. Exercising dyspnea with disease levels ranging from mild to severe (New York Heart Association Function Class III to IV) is often difficult to treat, leading to progressive disability. So, the early and accurate identification of risk factors for advanced HF have aroused widespread concern.

Studies have shown that many gene mutations cause HCM. So the current research mainly focuses on the effect of gene mutation on HF, including genetic status[7]and mutation dosage[8].At the same time, many studies have suggested that HCM patients with genetic mutations have worse symptoms and more severe adverse outcomes than those with negative mutations[9].Unfortunately, patients with negative mutations are ignored. Moreover, studies have found that the risk of poor prognosis remains in patients with negative genetic mutations[8].Therefore, it is urgent to study the prognostic factors of HCM patients without mutations and the influence of these risk factors.

In this study, we sought to analyze the correlation between the prognostic factors and the clinical phenotype in gene-negative patients with HCM, and evaluate the value of the factors in predicting the advanced HF, so as to provide a reference for the prognosis assessment of such patients.

\section{METHODS}

\section{STUDY POPULATION AND CLINICAL EVALUATION}

A total of 1529 unrelated patients with HCM were enrolled from 1999 to 2018 at Fuwai Hospital, Chinese Academy of Medical Sciences. Patients were diagnosed by a maximum left ventricular wall thickness $\geq 15 \mathrm{~mm}$ (or $\geq 13-14 \mathrm{~mm}$ if having a HCM family history) measured 
with 2-dimensional echocardiographic and/or cardiac magnetic resonance imaging, in the absence of other cardiac or systemic diseases capable of producing such magnitude of ventricular hypertrophy[10].Patients without mutations were included in the study. This study was approved by the Ethics Committee of Fuwai Hospital and the Affiliated Hospital of Qingdao University, and conformed to the principles of the Declaration of Helsinki. All participants gave written informed consent.

Clinical evaluation included sex, age at enrolment, family history of HCM, family history of SCD, syncope, left ventricular end-diastolic diameter (LVEDD), body surface area (BSA), left atrium (LA)diameter, maximal left ventricular wall thickness (LVWT), left ventricular ejection fraction (LVEF), systolic anterior movement (SAM), left ventricular outflow tract (LVOT) gradient and atrial fibrillation (AF).

\section{FOLLOW UP and OUTCOMES MEASUREMENT}

The patients were prospectively recruited and followed up by clinical visits or telephone consultation. The clinical outcomes measured in this study were advanced HF. We defined New York Heart Association class III or IV (NYHA III/IV) HF as advanced HF.

\section{STATISTICAL ANALYSIS}

Continuous variables, reported as means with mean \pm standard deviation (SD), were compared between groups with t-test or nonparametric tests, as appropriate. Multiple groups were compared using one-way ANOVA. Categorical variables, reported as number (\%), were compared between groups with $\mathrm{x} 2$ or Fisher exact tests. Survival was modelled using Kaplan-Meier analysis and log-rank test. Hazard ratios (HRs) and 95\% confidence intervals (CIs) were estimated with univariable or multivariable Cox proportional hazard regression models. All probability values were 2-sided, and $\mathrm{P}<0.05$ was considered significant. SPSS (V.23.0.0.0) was used for the analyses.

\section{Results}

\section{Characteristics of Patients}

A total of $735(48.1 \%)$ unrelated patients with HCM were included in this study following the inclusion and exclusion criteria. The mean age at enrolment the studied patients was $52.54 \pm 13.24$ years and $69.5 \%$ was male. The demographic and clinical characteristics of the studied patients were summarized in Table 1.

\section{Clinical Outcomes}


During the mean follow-up period of $3.18 \pm 2.28$ years, there were 95 advanced HF. The univariate analysis showed that gender, age at enrolment, BSA and AF were related to advanced HF events. The multivariate Cox regression analysis and Kaplan-Meier analysis revealed that female was associated with a significantly higher risk of advanced HF than male (adjusted HR $2.499,95 \%$ CI 1.531-4.081, $\mathrm{P}<0.001$ Table 2, Figure 1). The analysis also showed that older at enrolment (adjusted HR 1.298,95\% CI 1.000-1.682, $\mathrm{P}=0.049$, Table 2 Figure 1).

\section{DISCUSSION}

The presence of different phenotypes in any of the pathogenic mutations suggests that other factors besides genetic mutations may influence the development of HCM. And the 2014 guidelines of the European Society of Cardiology suggested that the cause of HCM in 25-30\% of patients remains unknown[11].In our study, we enrolled sarcomere gene mutation-negative HCM patients, and found that female and older at enrolment are associated with increased risk of advanced HF in HCM patients without mutations during the follow-up period.

HCM patients may have a high prevalence of HF. One of the studies, in a cohort of 1000 patients diagnosed with $\mathrm{HCM}$, showed a $\mathrm{HF}$ incidence of nearly $50 \%$, with symptoms ranging from mild to severe[12]. Another study, with a larger cohort of 3208 patients, showed that the prevalence of $\mathrm{HF}$ was $67 \%[13]$. Thus, early and accurate identification of risk factors for advanced HF is of great value for the treatment and management of patients with HCM.

Some biomarkers have been related to the disease severity of HCM, However, due to lack of sensitivity and specificity, they cannot be a reliable clinical tool to guide treatment. For example, Brain natriuretic peptide (or N-terminal pro-brain natriuretic peptide) may be useful for nonobstructive HCM patients, but it is not a liable prognostic predictor for individuals with HCM[14]. In the past 15 years, genetic testing has played an important role in guiding the clinical management of patients with HCM. Genetic testing maybe offer excellent opportunities to identify individuals with mutations who will develop HCM before typical clinical manifestations arise. However, there is insufficient evidence that sarcomere gene mutations can predict the development of HF in patients with HCM, although mutation-positive patients are associated with a worse cardiovascular prognosis than mutation-negative patients[15].

During the past 20 years, a large number of studies have been conducted on HCM patients of different groups and races to determine whether the prognosis of women is worse than that of 
men.[16-21]. Studies have found that patients with gene mutations have a worse prognosis than mutation-negative patients[8].After excluding the influence of genetic mutations on patients, we conducted research and found that women still can increase the risk of advanced HF during follow-up. This is consistent with previous studies ${ }^{[22]}$.This indicates that gender is an important determinant in HCM treatment, and women may need more aggressive diagnosis and treatment methods.

A major risk factor for $\mathrm{HF}$ and overall cardiovascular disease is age. About $1 \%$ of people over the age of 50 suffer from HF, which doubles every 10 years, making HF the leading cause of death for the elderly. [23].In the present study, HCM patients with sarcomere gene mutations are younger at enrolment[8]. It can be seen that gene mutations have an impact on the age at enrolment of patients with HCM. Thus, in the case of excluding this factor of gene mutation, our study found that older at enrolment was significantly increased the risk of advanced HF during follow-up. We warn that negative-mutation patients with HCM should still pay attention to the disease, and seek medical treatment in time after diagnosis and review them regularly to minimize the possibility of adverse events.

\section{CONCLUSION}

Our study demonstrates that gender and age at enrolment are predictor of advanced HF events in gene-negative patients with HCM. This study may have implications for the management of HCM patients without gene mutations.

\section{List of abbreviations}

\begin{tabular}{|l|l|}
\hline hypertrophic cardiomyopathy & HCM \\
\hline heart failure & HF \\
\hline hazard ratio & HR \\
\hline confidence interval & CI \\
\hline sudden cardiac death & SCD \\
\hline left ventricular end-diastolic diameter & LVEDD \\
\hline Body Surface Area & BSA \\
\hline left atrium & LA \\
\hline left ventricular wall thickness & LVWT \\
\hline
\end{tabular}




\begin{tabular}{|l|l|}
\hline left ventricular posterior wall & LVPW \\
\hline left ventricular ejection fraction & LVEF \\
\hline systolic anterior movement & SAM \\
\hline left ventricular outflow tract & LVOT \\
\hline atrial fibrillation & AF \\
\hline
\end{tabular}

\section{Declarations}

Ethics approval and consent to participate: This study conformed to the principles of the

Declaration of Helsinki. All participants gave written informed consent.

Consent for publication

Availability of data and materials: Not applicable.

Competing interests: The authors declare that they have conflicts of interest.

Funding: None.

Acknowledgements: The authors would like to thank all participants for their time.

Authors' contributions: Study conception and design: Lin Qi. Data collection: Zhengyi Shan, Ying Wei, Qingyao Wang. Data analysis and interpretation: Lin Qi. Drafting of the article: Lin Qi. Critical revision of the article: Lin Qi, Peng Zhao. All authors have read and approved the manuscript.

\section{Reference}

1. Marian AJ, Braunwald E. Hypertrophic Cardiomyopathy: Genetics, Pathogenesis, Clinical Manifestations, Diagnosis, and Therapy. Circ Res. 2017;121(7):749-70.

2. Maron BJ, Gardin JM, Flack JM, Gidding SS, Kurosaki TT, Bild DE. Prevalence of hypertrophic cardiomyopathy in a general population of young adults. Echocardiographic analysis of 4111 subjects in the CARDIA Study. Coronary Artery Risk Development in (Young) Adults. Circulation. 1995;92(4):785-9.

3. Zou Y, Song L, Wang Z, Ma A, Liu T, Gu H, et al. Prevalence of idiopathic hypertrophic cardiomyopathy in China: a population-based echocardiographic analysis of 8080 adults. Am J Med. 2004;116(1):14-8.

4. Maron BJ. Clinical Course and Management of Hypertrophic Cardiomyopathy. N Engl J Med. 2018;379(20):1977.

5. Rowin EJ, Maron BJ, Kiernan MS, Casey SA, Feldman DS, Hryniewicz KM, et al. Advanced heart failure with preserved systolic function in nonobstructive hypertrophic cardiomyopathy: under-recognized subset of candidates for heart transplant. Circ Heart Fail. 2014;7(6):967-75.

6. Pasqualucci D, Fornaro A, Castelli G, Rossi A, Arretini A, Chiriatti C, et al. Clinical Spectrum, Therapeutic Options, and Outcome of Advanced Heart Failure in Hypertrophic Cardiomyopathy. Circ Heart Fail. 2015;8(6):1014-21.

7. Olivotto I, Girolami F, Ackerman MJ, Nistri S, Bos JM, Zachara E, et al. Myofilament protein 
gene mutation screening and outcome of patients with hypertrophic cardiomyopathy. Mayo Clin Proc. 2008;83(6):630-8.

8. Wang J, Wang $\mathrm{Y}$, Zou $\mathrm{Y}$, Sun $\mathrm{K}$, Wang $\mathrm{Z}$, Ding $\mathrm{H}$, et al. Malignant effects of multiple rare variants in sarcomere genes on the prognosis of patients with hypertrophic cardiomyopathy. Eur J Heart Fail. 2014;16(9):950-7.

9. Lopes LR, Rahman MS, Elliott PM. A systematic review and meta-analysis of genotype-phenotype associations in patients with hypertrophic cardiomyopathy caused by sarcomeric protein mutations. Heart. 2013;99(24):1800-11.

10. Elliott $P$, Andersson B, Arbustini E, Bilinska Z, Cecchi F, Charron P, et al. Classification of the cardiomyopathies: a position statement from the European Society Of Cardiology Working Group on Myocardial and Pericardial Diseases. Eur Heart J. 2008;29(2):270-6.

11. Authors/Task Force m, Elliott PM, Anastasakis A, Borger MA, Borggrefe M, Cecchi F, et al. 2014 ESC Guidelines on diagnosis and management of hypertrophic cardiomyopathy: the Task Force for the Diagnosis and Management of Hypertrophic Cardiomyopathy of the European Society of Cardiology (ESC). Eur Heart J. 2014;35(39):2733-79.

12. Maron BJ, Rowin EJ, Casey SA, Link MS, Lesser JR, Chan RH, et al. Hypertrophic Cardiomyopathy in Adulthood Associated With Low Cardiovascular Mortality With Contemporary Management Strategies. J Am Coll Cardiol. 2015;65(18):1915-28.

13. Charron P, Elliott PM, Gimeno JR, Caforio ALP, Kaski JP, Tavazzi L, et al. The Cardiomyopathy Registry of the EURObservational Research Programme of the European Society of Cardiology: baseline data and contemporary management of adult patients with cardiomyopathies. Eur Heart J. 2018;39(20):1784-93.

14. Geske JB, McKie PM, Ommen SR, Sorajja P. B-type natriuretic peptide and survival in hypertrophic cardiomyopathy. J Am Coll Cardiol. 2013;61(24):2456-60.

15. Garcia-Pavia P, Vazquez ME, Segovia J, Salas C, Avellana P, Gomez-Bueno M, et al. Genetic basis of end-stage hypertrophic cardiomyopathy. Eur J Heart Fail. 2011;13(11):1193-201.

16. Ho HH, Lee KL, Lau CP, Tse HF. Clinical characteristics of and long-term outcome in Chinese patients with hypertrophic cardiomyopathy. Am J Med. 2004;116(1):19-23.

17. Wang Y, Wang J, Zou Y, Bao J, Sun K, Zhu L, et al. Female sex is associated with worse prognosis in patients with hypertrophic cardiomyopathy in China. PLoS One. 2014;9(7):e102969.

18. Nijenkamp L, Bollen IAE, van Velzen HG, Regan JA, van Slegtenhorst M, Niessen HWM, et al. Sex Differences at the Time of Myectomy in Hypertrophic Cardiomyopathy. Circ Heart Fail. 2018;11(6):e004133.

19. Meghji Z, Nguyen A, Fatima B, Geske JB, Nishimura RA, Ommen SR, et al. Survival Differences in Women and Men After Septal Myectomy for Obstructive Hypertrophic Cardiomyopathy. JAMA Cardiol. 2019;4(3):237-45.

20. van Driel B, Nijenkamp L, Huurman R, Michels M, van der Velden J. Sex differences in hypertrophic cardiomyopathy: new insights. Curr Opin Cardiol. 2019;34(3):254-9.

21. Geske JB, Ong KC, Siontis KC, Hebl VB, Ackerman MJ, Hodge DO, et al. Women with hypertrophic cardiomyopathy have worse survival. Eur Heart J. 2017;38(46):3434-40.

22. Rowin EJ, Maron MS, Wells S, Patel PP, Koethe BC, Maron BJ. Impact of Sex on Clinical Course and Survival in the Contemporary Treatment Era for Hypertrophic Cardiomyopathy. J Am Heart Assoc. 2019;8(21):e012041.

23. Benjamin EJ, Muntner P, Alonso A, Bittencourt MS, Callaway CW, Carson AP, et al. Heart 
Disease and Stroke Statistics-2019 Update: A Report From the American Heart Association. Circulation. 2019;139(10):e56-e528.

Table 1 Baseline clinical and echocardiographic findings of gene-negative HCM patients

\begin{tabular}{lllll}
\hline & Total & $\begin{array}{l}\text { Patients without events } \\
(\mathrm{n}=638)\end{array}$ & $\begin{array}{l}\text { Patients with events } \\
(\mathrm{n}=97)\end{array}$ & $\begin{array}{c}\text { P-values } \\
\end{array}$ \\
\hline Male, $\mathrm{n}(\%)$ & $511(69.5)$ & $464(72.7)$ & $47(48.5)$ & $<0.001$ \\
Age at enrolment, years & $52.54 \pm 13.24$ & $51.82 \pm 13.10$ & $57.29 \pm 13.25$ & $<0.001$ \\
Family history of HCM, n(\%) & $92(12.5)$ & $79(12.4)$ & $13(13.4)$ & 0.869 \\
Family history of SCD, $\mathrm{n}(\%)$ & $67(9.1)$ & $56(8.8)$ & $11(11.3)$ & 0.447 \\
Syncope, n(\%) & $68(9.3)$ & $58(9.1)$ & $10(10.3)$ & 0.707 \\
LVEDD, mm & $45.42 \pm 6.19$ & $45.37 \pm 5.80$ & $45.70 \pm 8.34$ & 0.713 \\
BSA & $1.92 \pm 0.25$ & $1.93 \pm 0.26$ & $1.86 \pm 0.21$ & 0.012 \\
LA diameter, mm & $40.70 \pm 6.58$ & $40.47 \pm 6.43$ & $42.14 \pm 7.33$ & 0.036 \\
Maximal LVWT, mm & $21.24 \pm 5.61$ & $21.08 \pm 5.69$ & $22.28 \pm 4.97$ & 0.051 \\
LVPW, mm & $11.67 \pm 2.72$ & $11.55 \pm 2.60$ & $12.46 \pm 3.33$ & 0.011 \\
LVEF, \% & $67.03 \pm 8.12$ & $67.40 \pm 7.59$ & $64.55 \pm 10.71$ & 0.013 \\
SAM, n(\%) & $322(43.8)$ & $286(44.8)$ & $36(37.1)$ & 0.187 \\
LVOT gradient, mm Hg & $43.25 \pm 43.42$ & $42.75 \pm 41.89$ & $46.54 \pm 52.47$ & 0.498 \\
AF, n(\%) & $84(11.4)$ & $65(10.2)$ & $19(19.6)$ & 0.010 \\
\hline
\end{tabular}

Values are $\mathrm{n}(\%)$ or mean $\pm \mathrm{SD}$.

HCM, hypertrophic cardiomyopathy; SCD, sudden cardiac death; LVEDD, left ventricular end-diastolic diameter; BSA, body surface area; LA, left atrium; LVWT, left ventricular wall thickness; LVPW, left ventricular posterior wall; LVEF, left ventricular ejection fraction; SAM, systolic anterior movement; LVOT, left ventricular outflow tract; $\mathrm{AF}$, atrial fibrillation.

Table 2Univariable and multivariable Cox regression analysis of the association between clinical characteristics and outcomes in patients with HCM

\begin{tabular}{lllll}
\hline Variants & Crude HR (95\% CI) & Crude P-value & Adjusted $^{\star}$ HR (95\% CI) & Adjusted $^{\star}$ P-value \\
\hline Male & $2.807(1.880-4.191)$ & $<0.001$ & $2.574(1.579-4.195)$ & $<0.001$ \\
Age at enrolment & $1.517(1.183-1.944)$ & 0.001 & $1.298(1.00-1.682)$ & 0.049 \\
BSA & $0.725(0.565-0.929)$ & 0.010 & $1.019(0.761-1.366)$ & 0.899 \\
LA diameter & $1.269(0.992-1.624)$ & 0.058 & - & - \\
LVEF & $0.798(0.624-1.021)$ & 0.072 & - & - \\
AF & $1.934(1.168-3.203)$ & 0.010 & $0.988(0.553-1.766)$ & 0.947 \\
\hline
\end{tabular}

${ }^{\star}$ Models were adjusted for male, age at enrolment, BSA, AF (follow up time)

BSA, Body Surface Area; LA, Left atrium; LVEF, left ventricular ejection fraction; AF, atrial fibrillation. 
A

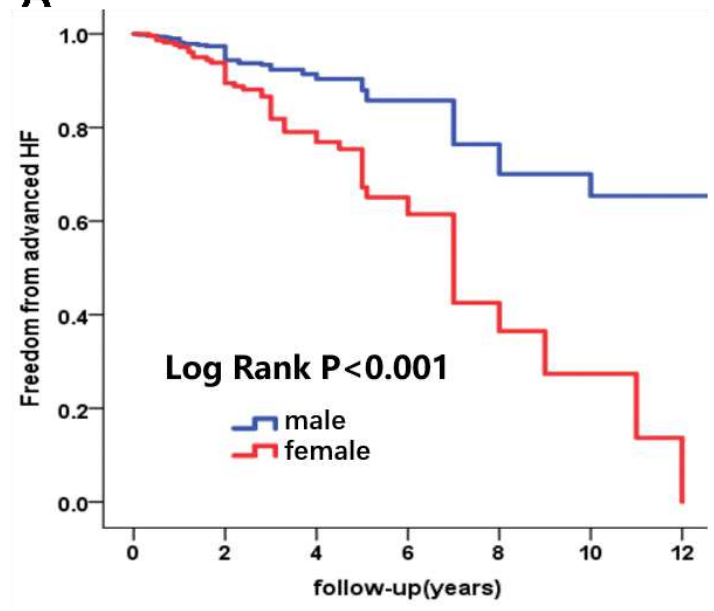

B

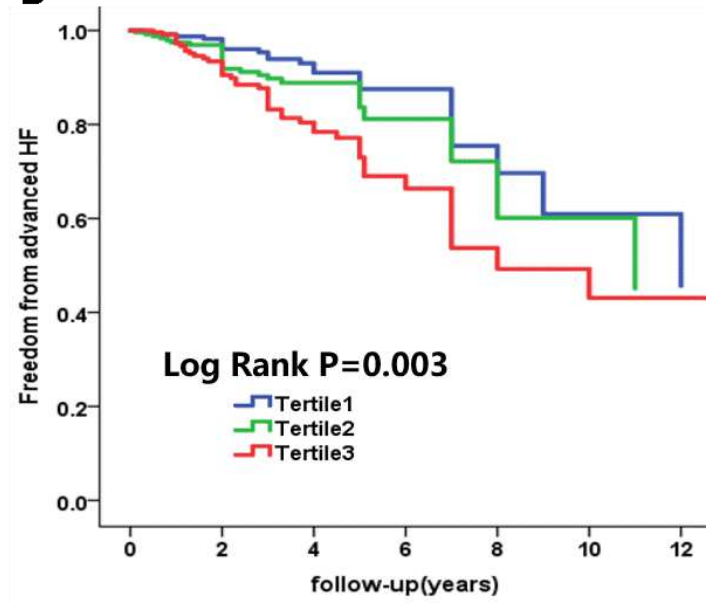

Figure 1 In follow-up time, survival curves for advanced HF events of genders(A), age at enrolment(B). The log rank test was used to calculate the $\mathrm{P}$ valu 
Figures
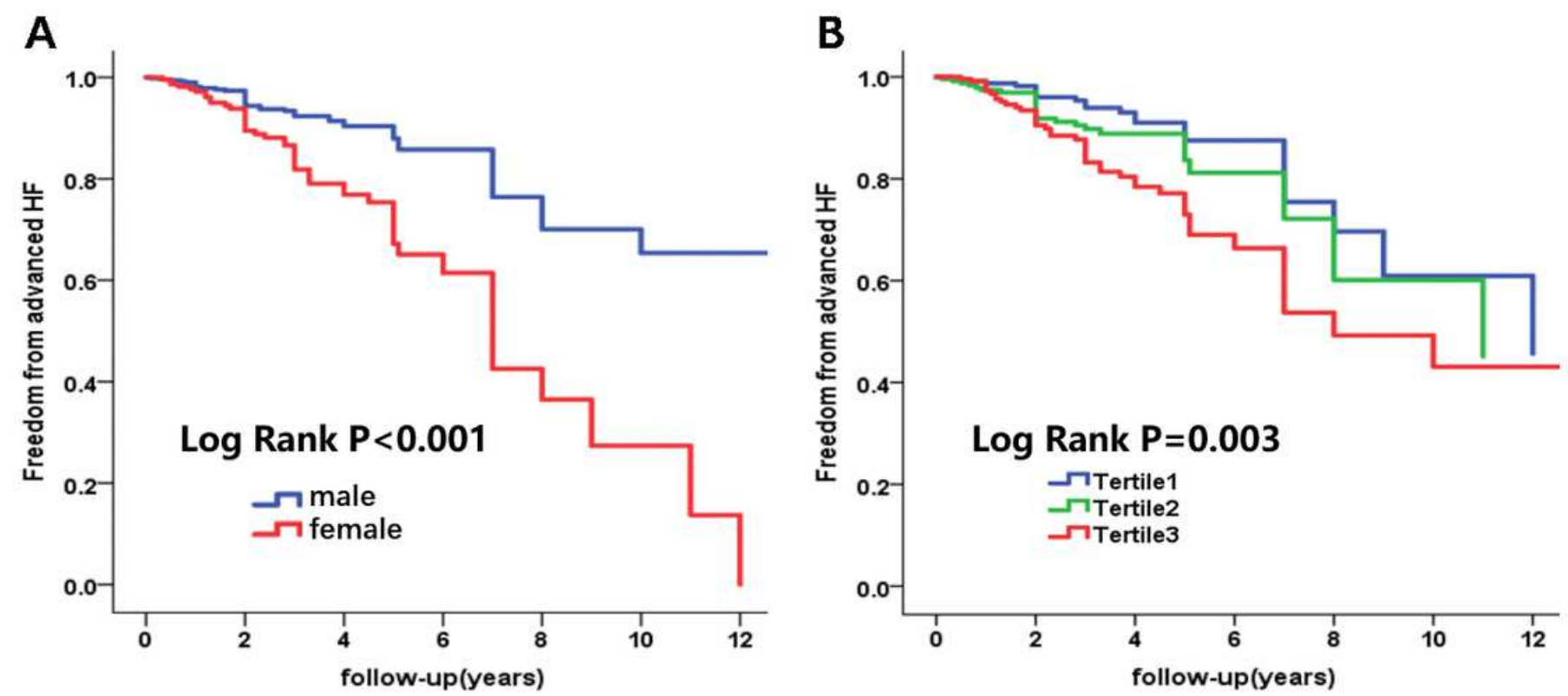

Figure 1

In follow-up time, survival curves for advanced HF events of genders(A), age at enrolment(B). The log rank test was used to calculate the $P$ value 